\title{
El estudio de los Pueblos Mágicos. UNA REVISIÓN A CASI 20 AÑOS DE LA IMPLEMENTACIÓN DEL PROGRAMA
}

\author{
Jesús Ángel ENRÍQuez ACOSTA \\ jesus.enriquez@unison.mx \\ Universidad de Sonora \\ ROSA YECENIA VARGAS OCHOA \\ yeceniav@yahoo.com \\ Universidad de Sonora
}

El Programa Pueblos Mágicos está próximo a cumplir 20 años de su diseño e instrumentación por parte de la Secretaría de Turismo en México. Hoy en día, 132 localidades tienen el nombramiento. El trabajo se propone hacer una revisión de los estudios realizados por académicos sobre Pueblos Mágicos, tomando en cuenta enfoques, casos, procedimientos y resultados. Se hizo un ejercicio de agrupación atendiendo a las temáticas más abordadas. En ese sentido, cinco grandes temas parecen predominar: política pública y turismo, turismo y patrimonio, impactos locales del turismo, imaginarios y representaciones del turismo, así como sustentabilidad y turismo.

Palabras clave: Pueblos Mágicos, política pública, patrimonio cultural, turismo, académicos.

\section{THE STUdY OF THE Pueblos MÁgICOS. A NEARLY 20-YEAR REVIEW OF THE IMPLEMENTATION OF THE PROGRAM}

The Pueblos Mágicos Program is close to celebrate 20 years of its design and implementation by the Ministry of Tourism in Mexico. Today, 132 towns have the title. This work aims to review the studies carried out by researchers about Pueblos Mágicos, taking into consideration approaches, cases, procedures and results. Grouping arrangement was carried out according to the most addressed topics. In this sense, five major subjects seem to prevail: public policy and tourism, tourism and heritage, local impacts of tourism, imaginary and tourism representations as well as sustainability and tourism.

Keywords: Pueblos Mágicos, public policy, cultural heritage, tourism, academics.

Fecha de recepción: 28 de septiembre de 2020 Fecha de aceptación: 15 de enero de 2021

CÓMO CITAR: Enríquez, J. y Vargas, R. (2021). El estudio de los Pueblos Mágicos. Una revisión a casi 20 años de la implementación del programa. Dimensiones Turísticas, 5(8), 9-38. https://doi.org/10.47557/SYWY9441 


\section{Introducción}

E I Programa de Pueblos Mágicos (PPM) surgió en el año 2001 por iniciativa de la Secretaría de Turismo (Sectur) del gobierno federal mexicano, con el objetivo de lograr un mayor desarrollo económico local, crear empleo y elevar el nivel de bienestar de las comunidades a partir de explotar con fines turísticos los atractivos naturales, patrimoniales y culturales de las localidades (Diario Oficial de la Federación, DOF, 2014).

Cercano a cumplir 20 años de existencia, parece un momento oportuno para realizar una revisión de los casos, enfoques, herramientas de análisis y resultados de los diversos estudios emprendidos por las comunidades académicas de México y el extranjero, las cuales han tenido como centro a los denominados Pueblos Mágicos (PM).

El estudio de los PM despertó gran interés en el ámbito académico, ante las implicaciones que tiene la política pública al denominar ciertos pueblos como singulares debido a los recursos y atractivos que ostentan para el turismo, además de sus pretensiones de posibilitar desarrollo local, involucrar a las comunidades y poner en valor a localidades que históricamente estuvieron exentas de las políticas de desarrollo turístico. Sin duda, también ocasionaron dicho interés los impactos y consecuencias del PPM sobre los sitios y territorios, lo que permitió reflexionar acerca del patrimonio tangible e intangible y las posibles amenazas del turismo.

Asimismo, propició analizar la aceptación o rechazo de la política por las comunidades y conocer la oferta turística basada en productos de tipo religioso, naturales, gastronómicos, arquitectónicos, fiestas y tradiciones, artesanías, entre otros aspectos (López et al., 2015). De ese modo, los PM fueron motivo de diversos estudios e investigaciones tendientes a revisar las singularidades enunciadas por el PPM y los nuevos retos que tales estudios arrojan en cuanto a dimensiones como gobernanza, sustentabilidad, imaginarios sociales, procesos de urbanización, tematización, entre otros (Madrid, 2019).

Antes de entrar a la revisión es preciso conocer un poco más acerca del PPM en cuestión. Se trata de una política pública al parecer aceptada por los gobiernos y comunidades participantes, así como por aquellas que pretenden su incorporación. Ha generado grandes expectativas debido a sus pretendidos beneficios locales, los turistas lo ven con buenos ojos y la marca PM dinamizó la oferta turística ante el dominante turismo de sol y playa característico de México. 
Actualmente, es una de las políticas de turismo que ha tomado gran relevancia para el gobierno mexicano, si consideramos su continuidad sexenal, la cobertura territorial por el número de localidades contempladas, su carácter intersectorial (ya que implica diversos niveles de gobierno y dependencias federales), así como estar sujeta a reglas de operación. En ellas se establecen los marcos de acción de los sectores involucrados, las condiciones hacia la obtención y pérdida del nombramiento para las localidades, formas de participación comunitaria, mecanismos de control y vigilancia de los recursos empleados, existencia de planes de desarrollo local y estatal, los recursos y atractivos turísticos de las localidades, entre otros puntos (DOF, 2015).

Los objetivos del PPM son: organizar una oferta turística basada en los atributos históricos-culturales de las localidades, generar productos turísticos referidos a las expresiones de la cultura local (fiestas, comida, artesanías, etcétera), fomentar el turismo de naturaleza o ecoturismo, poner en valor los recursos o atractivos turísticos de las localidades, entre otros (DOF, 2014).

La Sectur (2020a, párr. 1) refiere como PM a "un sitio con símbolos y leyendas, poblados con historia que en muchos casos han sido escenario de hechos trascendentes para nuestro país, son lugares que muestran la identidad nacional en cada uno de sus rincones, con una magia que emana de sus atractivos".

Considerando lo anterior, en cuanto a la valorización de la oferta turística, el PPM contribuye a "revalorar a un conjunto de poblaciones del país que siempre han estado en el imaginario colectivo de la nación y que representan alternativas frescas y variadas para los visitantes nacionales y extranjeros" (Sectur, 2020a, párr. 2). Con esta definición se puso en el orden del día de la investigación científica la reflexión en torno a entender los procesos de turistificación de localidades que históricamente permanecieron al margen de las políticas de turismo; además, sirvió para poner en cuestión al denominado turismo cultural y sus efectos en las comunidades, ayudó también a la discusión de las posibles amenazas y cambios que conlleva esta actividad sobre su patrimonio tangible e intangible, entre otros procesos.

A casi 20 años del origen del PPM, el actual gobierno federal (2018-2024) busca replantear la política pública estableciendo la Estrategia Nacional de Pueblos Mágicos (Sectur, 2020c). En este nuevo planteamiento se reconocen diversas limitaciones en la operación del PPM que no han incidido plenamente en el mejoramiento de las condiciones de vida de la población que habita los PM, o generado mayor desarrollo local, fomentado la participación social de las comunidades y constituido 
una real oferta turística, por vía de explotar el patrimonio histórico y cultural de las localidades.

En el diagnóstico incluido en la exposición de motivos de la nueva estrategia nacional se describen algunos procesos sociales, demográficos, económicos, servicios turísticos, entre otros, escenificados en los PM y que justifican la reformulación del PPM. La desigualdad, la precariedad social, desarrollo local limitado, insuficiente infraestructura turística y calidad cuestionable de los servicios afines son comunes en los PM.

Así, se señala que pese a la diversidad cultural y natural del país en solo 10 estados del país se concentra el $60 \%$ de los PM. En cuanto a la dinámica demográfica, en casi la mitad de los PM se registró un descenso de población, indicando con esto que la operación del PPM no es determinante para retener a los pobladores en las localidades.

Otro dato resaltante se refiere a las condiciones de marginación de los municipios considerados PM, en donde los niveles de pobreza y pobreza extrema son superiores a los promedios nacionales; aparentemente los beneficios económicos resultado de la turistificación de las localidades no tienen un alcance extensivo a los habitantes que viven con niveles de pobreza altos. Igual ocurre con el ingreso, el porcentaje de población que percibe ingresos inferiores a la línea de bienestar es alto y superior al promedio nacional. Los índices de desarrollo humano de los municipios son bajos, menores incluso a los promedios nacionales. Ese es el panorama general que ofrecen los PM en el presente.

\section{El estudio de los PM}

Parte de la comunidad académica interesada en el fenómeno del turismo, principalmente de universidades mexicanas, se orientó al estudio de los PM procurando analizar desde distintas miradas los problemas, dimensiones y procesos existentes en las localidades sujetas a la operación del PPM. Del interés mostrado por los investigadores a lo largo de casi 20 años del programa surgieron iniciativas muy importantes y valiosas para el estudio de los PM, de las cuales se desprendieron diversas publicaciones en artículos de revistas, libros y capítulos de libro. El estudio y las publicaciones en el tema son realizados en gran parte por esfuerzos colectivos de académicos y no por iniciativas individuales. Se resaltan dos ejemplos: 
El primero condujo a la formación de redes nacionales y foros de discusión. Destacable es la creación de la Red Ciudad, Turismo e Imaginarios, la cual integró investigadores de diversas universidades y tuvo como objetos de estudio a varios PM. Aunado a esto surgieron proyectos paralelos y complementarios a las temáticas de estudios de la Red, que permitieron la participación de académicos de iniciativas similares provenientes de diferentes ciudades mexicanas y de diversos países.

Estos proyectos representaron un parteaguas y fueron semilleros en la realización de nuevos esfuerzos, los cuales ampliaron los casos de estudio sobre el turismo en los PM y se plasmaron en la creación de la revista Topofilia y del Coloquio Ciudades del Turismo, importantes foros de análisis y reflexión académica sobre PM en México. Además, estas actividades incorporaron investigaciones de estudiantes a manera de tesis de doctorado, maestría y licenciatura.

Otro ejemplo notable es el empeño sostenido por académicos en la investigación tendiente a conocer y analizar a los PM. De llamar la atención es el trabajo realizado por investigadores de la Universidad Autónoma Metropolitana (UAM) y la Universidad Nacional Autónoma de México (UNAM) que condujo a la publicación de Pueblos Mágicos. Una visión interdisciplinaria, una colección de cuatro volúmenes en donde se hace un recuento pormenorizado de casi la totalidad de los PM y otras localidades interesadas en ingresar al PPM (López et al., 2014; 2015; 2017 y 2018). Por supuesto, existen otros casos exitosos de trabajos de investigación emprendidos en este periodo.

De los ejemplos de corte académico arriba citados y otros más no descritos por motivos de espacio, se destacan los distintos enfoques y perspectivas desde las cuales se estudia y reflexiona en torno a los PM; la interdisciplina es predominante en la investigación y la variedad de aproximaciones metodológicas emprendidas es la constante.

Los estudios efectuados tratan a la política pública desde diversos ángulos y es relevante la crítica realizada a la operación del PPM. De igual modo, se adentran en indicar los supuestos beneficios y contribuciones al desarrollo local, pero también las limitaciones y obstáculos presentados. Se discute ampliamente sobre las particularidades del patrimonio de las localidades y la puesta en valor de los atractivos turísticos. 
Por supuesto, al tratarse de una política pública que busca el desarrollo local mediante el turismo, son abundantes los estudios que comprenden los aspectos urbanos y territoriales, arquitectónicos, históricos, los impactos sociales y culturales, así como la sustentabilidad de la actividad turística en los PM.

Como es de esperar, los estudios igualmente abarcan las expectativas de las comunidades en relación con el PPM, así como los costos asociados a la instrumentación de la política, tales como: exclusión de la población local, segregación socioespacial, tematización y comercialización del patrimonio y los lugares, además de la gentrificación de algunos centros históricos.

\section{Metodología}

El objetivo del trabajo es realizar una revisión de las publicaciones académicas sobre PM surgidas en los últimos 12 años, tomando en cuenta las principales temáticas abordadas, enfoques considerados, los casos, procedimientos metodológicos y resultados. Se trata de una investigación exploratoria y descriptiva, haciendo análisis de contenido de las publicaciones para identificar las tendencias principales bajo las cuales los investigadores y colectivos de académicos en México han estudiado los PM.

Ante la imposibilidad de hacer un recuento de todos los trabajos académicos realizados en relación con los PM en las casi dos décadas de vida del programa, se hizo un ejercicio de agrupación de ellos atendiendo a las temáticas más abordadas. Durante el primer semestre de 2020 se efectuó una revisión en los buscadores Google Académico, Academia, Researchgate, Redalyc y Scielo; también se realizó una búsqueda de artículos publicados en la revista Topofilia como fuente conocida y de primera mano en la difusión de los estudios del turismo y PM. Igualmente, se recurrió a revistas como El Periplo Sustentable, PASOS, Investigaciones Turísticas, entre otras.

Asimismo, se consultaron libros académicos, principalmente publicados por universidades mexicanas, donde aparecieran capítulos orientados al estudio de los PM. Se seleccionaron los trabajos científicos publicados entre los años 2008 y 2021 en donde aparecieran en el título o en las palabras clave las referencias a PM y a conceptos asociados, tales como: patrimonio cultural, política pública, gobernanza, desarrollo local, identidad, imaginarios, representaciones, turismo cultural, imagen urbana, sustentabilidad, impactos y consecuencias locales, principalmente. 
Cada una de las referencias localizadas fue ordenada y clasificada de acuerdo con los criterios de búsqueda indicados. De esta manera, se agruparon por orientaciones disciplinarias y áreas temáticas con la finalidad de reunir los textos y encontrar ejes de estudio sobre PM. De igual forma, se organizaron los textos atendiendo a las herramientas metodológicas empleadas.

Se pudo constatar que las publicaciones recurren a técnicas cuantitativas, tratando de cuantificar variables de orden estructural y percepciones de grupos específicos; a la par, las técnicas cualitativas son de utilización recurrente y tratan de comprender subjetividades, valoraciones y significaciones acerca de dimensiones simbólicas, contextuales y paisajísticas. Finalmente, se destacan las publicaciones que utilizan técnicas mixtas.

La mayoría de los trabajos detectados son estudios de caso referidos a PM, se recurre al análisis de las localidades utilizando estrategias que van desde el cotejo documental, la realización de entrevistas en las comunidades, uso de la fotografía y mapas, consulta de archivos, levantamiento de encuestas, recorridos de observación en campo, entre otros recursos.

El trabajo de clasificación de acuerdo con los criterios de búsqueda permitió agrupar cinco grandes temas: a) política pública y turismo, b) turismo y patrimonio, c) impactos locales del turismo, d) imaginarios y representaciones del turismo y e) sustentabilidad y turismo. Los indicadores de cada uno de estos temas se obtuvieron a partir de la lectura de las publicaciones y el tratamiento específico de los procesos locales observados en los PM. Desde luego, se detectaron otras temáticas relacionadas con los PM, aunque el tratamiento fue tangencial o no era el eje central del análisis por lo cual no se incluyeron. Las publicaciones se organizaron en una tabla general por temas, estos dieron lugar a los ejes temáticos (ver tabla 1): 
Tabla 1. Agrupación de publicaciones por temas

\begin{tabular}{|c|c|}
\hline Ejes temáticos & Temas abordados \\
\hline \multirow{6}{*}{ Política pública y turismo } & Gobernanza \\
\hline & Participación ciudadana \\
\hline & Implementación de política pública \\
\hline & Evaluación de resultados \\
\hline & Desarrollo local \\
\hline & Cambios territoriales y urbanos \\
\hline \multirow{5}{*}{ Turismo y patrimonio } & Modalidades de turismo cultural \\
\hline & Patrimonio tangible e intangible \\
\hline & Conservación del patrimonio \\
\hline & Percepciones sobre el patrimonio \\
\hline & Tematización del lugar \\
\hline \multirow{6}{*}{ Impactos locales del turismo } & Costos y beneficios en las localidades \\
\hline & Amenazas al patrimonio \\
\hline & Desigualdad y desplazamiento \\
\hline & Gentrificación \\
\hline & Cambios en la vida local \\
\hline & Flujos de turistas \\
\hline \multirow{7}{*}{ Imaginarios y representaciones del turismo } & Narrativas locales \\
\hline & Reconfiguración del patrimonio \\
\hline & Homogenización cultural \\
\hline & Imaginarios de la promoción turística \\
\hline & Producción simbólica del lugar \\
\hline & Itinerarios turísticos \\
\hline & Imagen urbana \\
\hline \multirow{6}{*}{ Sustentabilidad y turismo } & Cuidado del medio y paisaje natural \\
\hline & Equidad social \\
\hline & Bienestar de la población \\
\hline & Tipos de desarrollo turístico \\
\hline & Propuestas de desarrollo sustentable \\
\hline & Protección del patrimonio cultural \\
\hline
\end{tabular}

Fuente: Elaboración propia. 


\section{Temáticas estudiadas}

Como resultado del trabajo de búsqueda y clasificación de publicaciones relacionadas con PM se localizaron 246 documentos, de los cuales 138 fueron capítulos de libro, 11 libros, 12 tesis de posgrado y 85 artículos en revistas. La mayoría de las publicaciones corresponden a capítulos de libro y, como se mencionó previamente, las colaboraciones son parte de esfuerzos colectivos de investigación más que trabajos de corte individual.

Las disciplinas que estudian los PM son predominantemente: geografía, urbanismo, arquitectura, sociología, antropología, comunicación y turismo. Parte importante de los textos se publicó entre los años 2014 y 2019 (70\%). Los procedimientos metodológicos empleados son cualitativos en su mayoría (75\%).

A continuación, se hará una breve descripción de algunos textos seleccionados para ilustrar el tratamiento de los PM de acuerdo con el ejercicio de agrupamiento. Es preciso señalar que no se trata de hacer un recuento de todos los trabajos sobre PM detectados, sino ejemplificar a partir de la lectura de contenidos de los casos estudiados cómo estos fueron abordados, bajo qué enfoques, cuáles procedimientos metodológicos fueron empleados y qué resultados se obtuvieron.

Por lo anterior, el ejercicio de agrupamiento que se presenta no tiene la finalidad de elaborar cuadros, gráficas o tablas donde se detalle y cuantifique la información detectada en forma de publicaciones, sino de comprender los énfasis y presupuestos que los académicos han dispuesto desde la investigación para el estudio de los PM. Puede asimismo existir una desigualdad o desequilibrio en cuanto al número de publicaciones referidas por cada uno de los ejes temáticos, pero ello se debe al mayor o menor desarrollo de líneas de conocimiento relacionadas con el estudio de los PM.

\subsection{Política pública y turismo}

La política pública federal y la local, al lado de inversiones privadas, permitieron consolidar determinados lugares turísticos denominados como PM, dotaron a las localidades de mayor infraestructura y equipamiento, alentaron las visitas de turistas ampliando la oferta de hospedaje y los servicios ligados al turismo, permitieron renovar la imagen urbana de los centros históricos, mejoraron las carreteras y la promoción de los lugares exaltando las peculiaridades de los destinos. 
La política pública facilitó que todo recurso paisajístico, patrimonial, simbólico e histórico, pudiera ser comercializable y puesto en valor para el turista. Ante este cambio en la fisonomía de los lugares, resulta pertinente valorar la hechura de dicha política pública y sus limitaciones en la instrumentación. En esta temática se puede encontrar una abundante y variada cantidad de textos, en donde predominan diferentes enfoques, énfasis y dimensiones consideradas.

Se analiza el PPM desde una revisión de su implementación y análisis de resultados en las localidades (Fernández, 2016) y se valora la eficacia de la política utilizando indicadores cuantitativos para medirla (Landeros et al., 2015). La gobernanza es un enfoque utilizado por Madrid (2014) así como Vázquez y Díaz (2015) para analizar los PM, la aptitud de la política de turismo, la articulación de lo público y lo privado en las localidades, a la par de los niveles de satisfacción de turistas y habitantes.

Otra línea interesante es el abordaje de las formas de participación ciudadana en términos de los niveles de involucramiento de la comunidad en la toma de decisiones (Muñoz, 2019). En la misma línea, se advierte la situación real de la población y el distanciamiento que existe entre la operación del PPM y la población local (Rojo y Llanes, 2009; Hernández, 2009). Álvarez-Quijada (2017) también analiza las percepciones de los habitantes de un PM con respecto a la implementación y gestión del PPM, a 10 años de su implementación, mediante la aplicación de una encuesta en la localidad.

Lara (2015) cuestiona la ejecución del PPM por no promover transparencia, rendición de cuentas y participación ciudadana; propone como alternativas la formulación de presupuestos participativos. De igual modo es importante el tratamiento de los PM a partir de comprender las contribuciones de la política al desarrollo local y los posibles fallos (Arévalo y Armas, 2019). Por su parte, Velázquez-García y Labra (2015) estudiaron los PM del Estado de Hidalgo realizando una evaluación de los resultados obtenidos por la política pública de turismo en las localidades.

Otro enfoque interesante es el utilizado por Vargas y Rodríguez (2014), quienes examinan un PM mediante el análisis de redes, los actores involucrados, las formas de participación, los tipos de gestión, entre otros. Ruiz (2016) utiliza la categoría de las prácticas y las relaciones construidas en la cotidianidad para analizar las formas en las que la población local se integra en el PPM, exponiendo tres tipologías principales: los que se involucran (dueños de servicios turísticos), los que participan (empleados) y los que se resisten o son excluidos por diferentes motivos. Encuentra que 
la percepción positiva o negativa de la población local sobre el PPM está en función de las relaciones construidas en el territorio.

Rodríguez (2013) argumenta que el PPM es una política reproducida, la cual ha mostrado efectos negativos que no son desconocidos por quienes la han implementado en territorio mexicano. La magia reconocida en los pueblos y la puesta en valor para el consumo tiende a desaparecer, por lo que identifica en la política pública desafíos y dilemas.

\subsection{Turismo y patrimonio}

La turistificación de localidades rurales y urbanas con el objetivo central de contribuir al desarrollo local, contrarrestar el olvido histórico de comunidades que fueron valiosas en otros momentos y diversificar la oferta turística, ante el declive de tradiciones productivas centradas en la agricultura y la minería, todo ello dio lugar a la puesta en valor del patrimonio cultural (tangible, intangible) con fines de desarrollar el turismo.

El PPM se inscribe en la línea del turismo cultural y con ello el patrimonio afín es sujeto de comercialización y venta turística. La política pública tiene como uno de sus objetivos principales estructurar una oferta turística complementaria y diversificada hacia el interior del país, en localidades con atributos histórico-culturales que les imprimen una notable singularidad. La representación de los PM refiere la imagen de localidades pequeñas, tranquilas, con población amable, llenas de folclor y tradición, sin las tensiones de la vida en las grandes ciudades y con un rico patrimonio cultural para ser consumido por los turistas.

Por supuesto, estas particularidades generaron bastantes reflexiones y polémicas en el medio académico. Las discusiones plantean que el PPM ofrece hoy fuertes retos para el equilibrio entre la valorización y conservación del patrimonio cultural, a la par de la práctica del turismo cultural (Enríquez et al., 2018); esto es, equilibrar los logros de tipo económico (de la empresa turística y su impacto local) y la preservación, protección y difusión del patrimonio cultural de las localidades.

Bajo ese supuesto, López et al. (2014; 2015; 2017 y 2018) acometieron la empresa de abordar prácticamente la totalidad de los PM. Cada uno de ellos pretende dar cuenta de los PM a partir de caracterizar el lugar, reseñar el patrimonio que ostenta y que justifica el nombramiento oficial, las relaciones sociales establecidas, el proce- 
so de construcción de imaginarios locales, las dinámicas del turismo, los impactos y beneficios de la operación del PPM, entre otros aspectos.

Martínez y Mínguez (2015) relacionan el patrimonio religioso de un PM referido a arquitectura y fiestas tradicionales con la política turística, en este caso el turismo religioso es la novedad. En la misma tónica, Enríquez et al. (2017) trabajan el caso de otro PM del noroeste de México, en donde el turismo religioso se mezcla con la devoción a un santo, desdibujando la diferencia entre turista y peregrino.

Manjarrez (2017) analiza la relación entre patrimonio y turismo cultural en PM y la ciudad de Puebla como Patrimonio Cultural de la Humanidad. En el texto reflexiona sobre la posibilidad de la coexistencia entre hacer turismo cultural, conservar el patrimonio y promocionar las localidades.

Aragón et al. (2016) indagan respecto a las implicaciones de la puesta en valor del patrimonio para el consumo turístico, muestran cómo las acciones implementadas por el PPM generan por una parte nuevas narrativas de la ciudad, en función de un imaginario turístico de pueblo, mientras por otra segregan los lugares y su población más vulnerable. Argumentan que al utilizar el patrimonio como atractivo turístico el valor imaginario se pierde, al construirse la imagen para el consumo, evaporándose así el imaginario local que lo constituía. Lo anterior se expresa analizando un PM colonial del noroeste de México.

Enríquez y Guillén (2017) se adentraron en la observación de los procesos de construcción de identidad y sentido de lugar en la misma población, enlazados en los elementos materiales y simbólicos de la localidad desde quienes la han construido, sentido y significado a lo largo de la historia, adentrándose también en las percepciones de los habitantes a través de las tramas narrativas construidas con respecto a su patrimonio cultural y su puesta en valor para el consumo turístico, identificando un sentido de pérdida en el relato.

Valenzuela et al. (2012) analizan la puesta en valor del patrimonio cultural en el PM de Tepoztlán y su incidencia en la identidad territorial, estableciendo así una distinción entre sus pobladores y la convivencia de dos imaginarios distintos sobre el mismo lugar, lo cual genera conflicto y resistencia por la población local, que busca reapropiarse del espacio del PM. 


\subsection{Impactos locales del turismo}

En parte importante de los estudios realizados sobre PM se observa que los beneficios del turismo son palpables en la economía local y en el mejoramiento de la imagen urbana, infraestructuras y equipamientos, de allí el interés de los gobiernos locales por que continúe el PPM y, más aún, se amplíe el número de comunidades integradas. Sin embargo, los costos de la actividad son igualmente visibles.

En el balance de estudios realizados en esta temática no se advierte una distribución con equidad de costos y beneficios; por el contrario, la depredación del paisaje urbano y natural, la acentuación de conflictos urbanos y sociales, los niveles de pobreza existentes, la percepción ciudadana lejana y desconectada del PPM, el encarecimiento de la vida, la especulación inmobiliaria, las amenazas al patrimonio, entre otros, hacen del turismo en los PM una actividad de la cual se podría cuestionar su sustentabilidad.

Con un enfoque cuantitativo, Cornejo et al. (2018) describen las percepciones que la población del PM de Tapalpa tiene sobre los impactos del turismo, se revela que las impresiones respecto a costos y beneficios no son claras y sí indistintas. Rodríguez (2017) revisa el impacto del PPM, a partir de utilizar datos socioeconómicos que permitieran conocer el antes y el después de la aplicación de la política pública. Concluye que es muy relativo el cambio en cuanto a la mejoría de condiciones de marginación social, empleo y pobreza de las localidades integradas al PPM.

Con ese mismo enfoque, Rojo y Castañeda (2012) valoran los efectos del programa en un PM del noroeste de México, realizando un balance desde los inicios del PPM y los cambios experimentados a cinco años de su implementación, mostrando la necesidad de planear un desarrollo turístico en apoyo a la localidad y con el apoyo de sus habitantes para lograr un equilibrio.

En este aspecto, Chavoya et al. (2013) analizan los procesos urbanos que han alterado el patrimonio natural y cultural en tres PM de la sierra de Jalisco, argumentando sobre la necesidad de un modelo urbano alternativo que sea armónico con el medio ambiente, que diversifique la economía local y genere bienestar local, al mismo tiempo que funja como instrumento de sustentabilidad.

En la misma línea, Baños et al. (2016) realizaron un estudio en dos PM, uno de Jalisco y otro de Nayarit. Identificaron los elementos del patrimonio local a través del rela- 
to de las significación e imaginarios y mostraron cómo estos son puestos en valor para la actividad turística y el desarrollo regional, encontrando que los beneficios de esta valoración no generan bienestar social colectivo y transgreden la identidad y valores locales.

Gómez et al. (2018) ponen en escena el pueblo de Bacalar, donde el imaginario turístico residencial transforma el paisaje natural, monta de manera forzada una imagen de PM para especular con el suelo, apropiarse de tierra ejidal y contribuir al despojo de la localidad. Balslev y Velázquez-García (2010) señalan la presencia de una comunidad de norteamericanos habitando en un PM del noroeste de México y las pugnas simbólicas escenificadas en el lugar, a la luz de un proceso de desplazamiento y gentrificación.

Vinculado al patrimonio, Valenzuela (2017) estudia la revalorización del suelo urbano y del mercado inmobiliario privado, al parejo de la turistificación del patrimonio cultural de un PM; el resultado es que la identidad comunitaria y el bienestar pretendido de la localidad quedaron de lado, frente a la consolidación de procesos de especulación realizada por capitales privados beneficiarios del turismo.

Córdova et al. (2018) ponen en duda la contribución de la política pública para mejorar las condiciones de vida de la población y generar desarrollo local. Encuentran que los jóvenes de un PM tienen escasas oportunidades de empleo en los variados servicios turísticos y deben migrar a otro lugar.

A este respecto, Noriega (2017) analiza los beneficios y consecuencias del PPM desde una perspectiva laboral. Ello muestra que a pesar de un aumento considerable de la participación local en la actividad turística, las condiciones laborales son las mismas en aquellos que se dedican a la actividad tanto formal como informal. Sin embargo, se reconoce un cambio en la apreciación y una distinción de aquellos dedicados a los servicios formales sobre los informales.

Enríquez (2018) y Enríquez y Guillén (2018) exploran diversos PM en donde la actividad turística es importante; concluyen que la población percibe de forma positiva al turismo, no así los beneficios en las localidades ni en las personas. Se distingue que el desarrollo del turismo solo es exclusivo de las empresas hoteleras y servicios turísticos; los ciudadanos están preocupados por los costos y amenazas probables al patrimonio e identidad de las comunidades. Otra perspectiva que aporta al estudio de los impactos del turismo es la de García (2017), quien revisa el paisaje urbano 
del PM de Mazamitla y observa un mejoramiento de imagen del espacio edificado, pero cuestionable en la preservación y conservación del patrimonio.

Desde la perspectiva del fenómeno urbano, Babini et al. (2012) analizan e interpretan el PM de Taxco en proceso de transformación económica a partir de la actividad turística. Argumentan que la intervención ha implicado cambios en su estructura urbana, en la forma de vida de sus habitantes, sus hábitos, costumbres y tradiciones, en la condición y funcionamiento del pueblo; además, tales procesos han contribuido al deterioro ambiental. Ello ha dificultado el ordenamiento y asignación de infraestructura y servicios, creando problemas de segregación, pobreza y violencia en la localidad.

Aunado a lo anterior, Alcaraz y Salgado (2012) señalan que el deterioro urbano producido por el proceso de turistificación ha sido también provocado por la falta de consenso en la construcción de un proyecto urbano integral sustentable y participativo para la conservación y rehabilitación del patrimonio, que además sea colectivo y no solo exclusivo del centro histórico, lugar atendido principalmente por el PPM.

\subsection{Imaginarios y representaciones del turismo}

En esta temática es sobresaliente el intento por narrar los PM a partir de los relatos de habitantes y turistas, referidos a las historias, leyendas y mitos construidos en torno a los lugares; la deconstrucción de la trama existente permite poner en claro al sitio o dar sentido a los sueños y fantasías, pero también a los temores y miedos. Se trata de un enfoque centrado en los imaginarios sociales de las localidades.

Bajo esa perspectiva, Alvarado et al. (2016; 2017), reúnen una serie de estudios sobre PM en Sonora, Sinaloa, Guerrero, Morelos, Puebla, Jalisco y Veracruz. El eje central son los lugares, los relatos orales y visuales de sus habitantes, los imaginarios locales construidos para representar identidades y pertenencias a los sitios.

Se analizan los imaginarios oficiales que pugnan por revalorizar la arquitectura colonial (Méndez y Rodríguez, 2013), los símbolos, las tradiciones, la historia local, mediante escenografías y montajes producto de las intervenciones de la política pública para mejorar y homogenizar la imagen urbana y que simulan un pasado glorioso, señorial, o bien engrandecer discursivamente las culturas locales de raíz indígena cotidianamente marginadas. 
En esta misma línea, Méndez y Baños (2013) atienden dos casos de estudio: Álamos, Sonora, y San Sebastián del Oeste, Jalisco, mostrando evidencias de cómo la arquitectura de estos pueblos ha sido intervenida para configurar múltiples representaciones de "lo mexicano", de acuerdo con el imaginario de turistas y promotores, en su puesta en valor como atractivos turísticos, logrando fortalecer dicha imagen sobre el imaginario original.

García y Méndez (2018) analizan la puesta en valor del patrimonio material e inmaterial en los PM a fin de explotarlos para el turismo, específicamente de la arquitectura. Observan que la narrativa asociada a la marca PM se constituye en un imaginario que homogeniza a las localidades y niega la diversidad. La magia de los lugares se diluye frente a la comercialización de la marca.

Zárate et al. (2018) examinan la oferta turística de un PM fronterizo a partir de los elementos turistificados y vislumbran las transformaciones, haciendo referencia a los impactos positivos y negativos de las intervenciones. Enfatizan que la imagen construida para el turismo no corresponde a la historia del lugar, por lo que la situación resulta contradictoria en cuanto a que la política busca rescatar las singularidades en los pueblos. Esta situación ha impactado en la identidad de los habitantes, aunque se reconoce que las intervenciones han sido significativas en cuanto a la derrama económica por la visita de turistas.

En ese sentido, Vargas y Enríquez (2015) estudian los imaginarios construidos por la promoción turística del PPM, que subsumen y desplazan a los imaginarios locales. De forma parecida, Rodríguez (2014) toma como caso al PM de Rosario, Sinaloa, y analiza los montajes realizados por el PPM sobre el patrimonio arquitectónico para valorizar el lugar y promoverlo. Igualmente, Velázquez-García y Aragón (2015) se acercan al PM de Todos Santos, Baja California Sur, para observar la construcción de las representaciones de "lo mexicano" de una comunidad de norteamericanos establecida en el lugar y las actividades turísticas desarrolladas a partir del imaginario evocado por el Hotel California.

Meza et al. (2018) estudian los elementos patrimoniales referidos a la muerte en su análisis de los PM. Bajo esa óptica, sugieren que los imaginarios de la muerte son atractivos para el denominado turismo oscuro. En otro aspecto, el trabajo de Enríquez et al. (2017) se enfocó en mostrar las posibilidades de desarrollo turístico que tiene el PM de Álamos, desde el imaginario de la población local. 
Sugerente es el trabajo de Fernández (2014) quien siguiendo la veta de los imaginarios se adentra en las emociones del turista y las identidades del habitante, hacia la configuración del turismo en un PM. Díaz y Osorio (2021) analizan la producción simbólica de un lugar turístico apropiado por diversos actores sociales, quienes intervienen mediante sus imágenes y experiencias en la transformación de las relaciones sociales y de los sujetos que habitan el PM.

En la temática de los imaginarios sociales, Alvarado et al. (2017) estudiaron diversos PM procurando rescatar saberes locales que contribuyeran a visibilizar el PM recordado, vivido e imaginado y con esto crear entornos más habitables para quienes han construido una relación con la localidad, pero también un PM más atractivo para el turismo, recobrando la singularidad, la magia del lugar difuminada o desplazada por las intervenciones, así como los imaginarios turísticos dominantes. En ese sentido, se buscó rescatar las voces de las habitantes, para lo cual se recurrió al imaginario local, a los imaginarios urbanos y turísticos de quienes han construido una relación con la localidad desde sus experiencias y prácticas.

Con esa misma óptica, Méndez (2016) interpreta el PM de Álamos a partir del mapa de los dispositivos locales, lugares, emblemas, intersticios, mesetas, tinglados, itinerarios, en la versión de los imaginarios locales, sin dejar de lado el pueblo reconstruido en la memoria y representado en mapas mentales, construyendo mapas de lugares que hacen legible el PM para quien lo recorre y relata.

Algunos trabajos hacen alusión a la magia del lugar en cuanto atractivo turístico que se pretende valorar por el PPM, tal es el caso de Valverde y Enciso (2013), quienes cuestionan si dicha magia es un atributo intrínseco, una designación o un decreto, en cuanto a que el PPM solicita cumplir con una serie de requisitos y un proceso de intervención del paisaje local para adquirir la cualidad mágica y la nominación de PM.

En esa misma línea, Fernández et al. (2013) indagan sobre qué es la magia de los PM desde el imaginario local de sus habitantes, para después proponer esquemas de planeación donde domine la voz de la comunidad. Fernández (2016) se centró en identificar cómo verbalizan la magia del PM Huasca de Ocampo en el imaginario social, tanto habitantes como visitantes, encontrando que el imaginario de tranquilidad está asociado a la magia del lugar presente en ambos relatos.

En continuidad con lo anterior, Babini y Hernández (2013) describen lo mágico de Taxco recuperado en los relatos de promotores y turistas; al mismo tiempo, mues- 
tran la realidad e inquietudes de sus habitantes en cuanto a la conservación de esa cualidad y la necesidad de considerar los sueños y anhelos de los pobladores en los proyectos turísticos.

Gabriela Rodríguez (2012) realiza un recorrido por Álamos en busca de la magia, identificando elementos en el espacio físico y reconociendo a través de estos el pueblo imaginado desde la experiencia individual. Concluye que ese encantamiento supuesto está en el ojo de quien mira el PM, pero de igual forma se construye con la intencionalidad del consumo.

Méndez y Sandoval (2013) muestran que la magia está guardada en los atributos culturales del imaginario de "lo mexicano" que se encuentra en los pueblos; su puesta en valor para el turismo implica reinventarlos para presentar su encanto. Al mismo tiempo muestra cómo es construida y verbalizada esa cualidad, desde la construcción del lugar en las voces de sus residentes y nativos.

Enríquez et al. (2015) llevan a cabo un acercamiento a los imaginarios sociales de los habitantes del PM El Fuerte para rescatar en ellos lo que la comunidad quiere, desea, y sueña de su PM, esto con la intención de pensar en acciones alternativas que reduzcan el distanciamiento existente entre la comunidad y el PPM.

Méndez (2012) da lectura a dos PM del noroeste de México para elaborar el mapa de lugares de la comunidad y ofrecer apuntes de la singularidad local, más allá de las miradas oficiales y promoción turística. Babini y Hernández (2012) se dan a la tarea de indagar en el imaginario histórico urbano y social de Taxco, a través de la recopilación fotográfica, dibujos, grabados y textos de diferentes fuentes y miradas, con la intención de valorar y a la par confrontar el imaginario turístico de la puesta en valor del patrimonio, para construir una propuesta de conservación y diversificar el turismo.

Valenzuela et al. (2012) rescatan el PM recordado, vivido y significado de actores que hasta el momento habían estado silenciados o excluidos en el proceso del rediseño del PM, a través de la técnica del dibujo aplicada a niños de primaria y jóvenes de la comunidad. Asimismo, en cuanto al abanico de posibilidades relacionadas con la oferta turística potencial desde los imaginarios sociales de los habitantes locales, Romero (2012) da lectura a un PM del noroeste de México y describe un itinerario turístico posible, partiendo del imaginario colectivo de sus habitantes como atractivo turístico potencial. 
Sylvia Rodríguez (2012) muestra recorridos turísticos alternativos recurriendo al imaginario colectivo de personajes nativos, guías turísticas locales de un PM, en contraposición con los construidos en el discurso empresarial y gubernamental. Rodríguez y Vargas (2013) analizan un fragmento del sistema turístico mexicano de pueblos entrelazado en el noroeste de México, mostrando algunos recorridos consumidos por los visitantes y el surgimiento de nuevas rutas construidas por el imaginario local, las cuales incluyen lugares no considerados en el mapa turístico de los PM.

\subsection{Sustentabilidad y turismo}

Los estudios realizados por académicos en donde se observa la vinculación entre PM y sustentabilidad son destacables (Alcázar y Olmos, 2020). Se piensa hasta qué punto el turismo es una actividad que cubre las necesidades tanto del turista como de la comunidad, conserva el patrimonio cultural y natural de las localidades, además de brindar condiciones para el desarrollo futuro de las localidades.

En ese sentido, los estudios son críticos en cuanto a presentar un panorama adverso para las localidades respecto a prácticas turísticas que amenazan el patrimonio y el paisaje, así como el medio natural. Cuestionan la mejora de condiciones de vida de la población anfitriona y enuncian fallos en la aplicación de la política pública, entre otros procesos; estas dinámicas no posibilitan un desarrollo turístico sustentable.

Es de reconocer que el actual gobierno federal destaque el valor e importancia de la sustentabilidad en el turismo practicado en los PM. La convocatoria emitida recientemente por la Sectur (2020b) para otorgar nuevos nombramientos establece como parte del proceso de registro que las localidades deberán contar con un ordenamiento referido al turismo sostenible, en particular a la conservación y regeneración del patrimonio.

Palafox et al. (2016) analizan un PM cuestionando la sustentabilidad social del turismo, ponen atención a la nueva ruralidad institucionalizada por el Estado y al proceso de debilitamiento de las actividades económicas tradicionales y de carácter primario de la localidad, frente a la turistificación observada como imposición.

Warnholtz (2014) cuestiona el PPM, analizando sus reglas de operación y los objetivos establecidos por no contribuir al desarrollo sostenible de las localidades a través del turismo, sino al contrario priorizar el avance turístico a costa del desarrollo sociocultural y ambiental. 
Ibarra y Velarde (2016) estudian un PM de Sinaloa donde determinadas prácticas turísticas relacionadas con el turismo de aventura dañan el patrimonio local. Los turistas congestionan calles, vandalizan el lugar y la sustentabilidad del turismo se pone a prueba. Otro trabajo interesante es el de Arista et al. (2021), quienes abordan la capacidad de resiliencia de dos PM para enfrentar o mitigar una crisis determinada, frente al desarrollo turístico y la aplicación de la política pública. Encuentran que la inclusión o exclusión del nombramiento PM puede generar desequilibrios fuertes en la conservación del patrimonio, crear mayor o menor vulnerabilidad socioeconómica de la población de acuerdo con la permanencia en el PPM y, por consiguiente, incidir en la sustentabilidad del turismo y de las localidades.

Equihua et al. (2015) hacen una crítica al PPM cuestionando que el mejoramiento de la imagen urbana de las localidades no significa sustentabilidad, piensan que los beneficios obtenidos no son duraderos y sólidos ni tampoco suscitan participación social. Saiz (2018) estudia el caso del PM de Tequila en Jalisco utilizando el análisis FODA, concluye que las industrias tequilera y turística se han beneficiado de la denominación de origen de su producto principal y el cuidado del ecosistema agavero; sin embargo, no ha tenido un efecto importante en abatir los niveles de pobreza de las localidades por la concentración de la riqueza en pocas manos, los monopolios tequileros existentes y la escasa diversificación económica, procesos que limitan la sustentabilidad de la industria turística.

Alvarado et al. (2017) ponen atención a las acciones realizadas por el programa en el PM de Tepoztlán, las cuales resultan no acordes con el desarrollo sustentable por solo considerar el área histórica de la localidad, donde se registra la mayor afluencia turística, para dejar en el olvido al resto de la comunidad.

Cazal y Omar (2015) cuestionan la sustentabilidad del PPM al observar el caso de Bacalar, donde la turistificación del lugar amenaza el patrimonio natural y el paisaje singular. García y López (2018) estudian un PM del Estado de Puebla y procuran identificar áreas de oportunidad referidas al desarrollo turístico sustentable, estableciendo vínculos entre este, la promoción turística, los problemas observados en las localidades y una agenda que prioriza el estudio de la sustentabilidad en los PM.

Shaadi et al. (2018) hacen una revisión del PPM a partir de estudios diagnósticos realizados por la Sectur, con datos referidos a la competitividad y sustentabilidad para obtener el modelo de ciclo de vida de los PM. Encontraron que la mayoría de las localidades están en fase de desarrollo y son pocas las consolidadas. 


\section{Conclusiones}

El diagnóstico realizado por el actual gobierno federal (Sectur, 2020d), coincide en gran medida con los resultados de las investigaciones realizadas por académicos anteriormente referidas. Como es de notar, la desigualdad, la precariedad social, la vulnerabilidad de las comunidades, escaso desarrollo local, deficiencias en la operación del PPM, amenazas al patrimonio por prácticas turísticas nocivas, beneficios exclusivos para ciertos grupos económicos, debilidades institucionales de los municipios y limitaciones hacia la participación ciudadana, son pautas generales en los PM y en el abordaje de los académicos en sus publicaciones.

En la revisión de los estudios académicos sobre PM prevalece un sentido crítico acerca de la política pública; no obstante, es posible observar y comprender algunos de los problemas propios de los PM, surgidos en parte de la operación de tal política, así como de los beneficios palpables en las localidades. Es de reconocer la intervención del PPM para mejorar la imagen urbana, rehabilitar el espacio construido, preservar el patrimonio e instalar infraestructuras urbanas básicas, aunque en la mayoría de las ocasiones se ha circunscrito a los centros históricos y tocado poco a las áreas fuera del primer cuadro de las localidades.

De igual modo, es acertado el PPM en cuanto a la revalorización del patrimonio cultural, preservar tradiciones e identidades locales, exaltar recursos gastronómicos y artesanales, entre otros. No obstante, también la puesta en valor del patrimonio lo somete a la amenaza de la comercialización y venta, a prácticas turísticas que lo destruyen, a la tematización cultural que no distingue diferencias entre las localidades y a la construcción de un imaginario de los PM como mera mercadotecnia turística.

Importante es destacar la contribución del PPM al desarrollo local, creando empleo, diversificando la economía, mejorando los servicios turísticos y elevando las condiciones de vida a poblaciones y localidades muchas veces pobres y marginadas. Pero también ha motivado la crítica a los beneficios, pues muchas veces son exclusivos de ciertos grupos sociales o económicos vinculados al turismo, excluyendo o desplazando a las comunidades, ocasionado especulación inmobiliaria y del suelo urbano.

Cuestionable resulta que un programa presuntamente dirigido a incrementar la participación de los diversos sectores sociales para socializar los beneficios, igual- 
mente sea percibido distante y ajeno a las dinámicas locales. Por supuesto, esto se agrava cuando el programa en muchos casos no incita a la participación de las comunidades, al no informar, consultar o convocar para tomar decisiones sobre lo que les afecta o beneficia.

\section{Referencias}

Alcaraz, O. y Salgado, A. (2012). Deterioro urbano de Taxco de Alarcón: pueblo mágico. Topofilia, Revista Científica de Arquitectura, Urbanismo y Territorios, 3(2), 1-22. https://bit.ly/3usEJIR

Alcázar, A. y Olmos-Martínez, E. (2020). Estado del conocimiento sobre el desarrollo sustentable en Pueblos Mágicos. Dimensiones Turísticas, 4(7), 93-124. https://doi.org/10.47557/OFXE8035

Alvarado, C., Babini, A., Baños, J., Cabrera, V., Guillén, M., Méndez, E., Rojo, S. y Zacarías, P. (Coords.). (2016). Pueblos Mágicos. Narrativas varias. Pearson Educación y Universidad Autónoma del Estado de México.

Alvarado, C., Babini, A., Baños, J., Guillén, M., Méndez, E., Rojo, S. y Velázquez-García, M. A.(Coords.). (2017). Lugares de encuentro. Una visita a pueblos mexicanos. El Colegio del Estado de Hidalgo. https://bit.ly/3qO79EJ

Alvarado, C., Ruiz, C., Valenzuela, A. y Calleja, M. (2017). El programa Pueblos Mágicos y el desarrollo sustentable en Tepoztlán. En J. Enríquez, M. Guillén y B. Valenzuela (Coords.), Patrimonio y turismo. Un acercamiento a los lugares turísticos de México (pp. 175-199). Universidad de Sonora y QARTUPPI. https://bit.ly/3mMQPT5

Álvarez-Quijada, R. (2017). Percepción ciudadana sobre el programa de Pueblos Mágicos. Estudio estadístico en Álamos, Sonora. En J. Enríquez, M. Guillén y B. Valenzuela (Coords.), Patrimonio y turismo. Un acercamiento a los lugares turísticos de México (pp. 69-86). Universidad de Sonora y QARTUPPI. https://bit.ly/3mMQPT5

Aragón, M., Méndez, E. y Velázquez-García, M. A. (2016). Turismo y patrimonio. Esbozo del "pueblo mágico" Álamos. Topofilia, Revista Científica de Arquitectura, Urbanismo y Territorios, 9(13), 3-16. https://bit.ly/3pCNwhA 
Arévalo, J. y Armas, E. (2019). Pueblos mágicos: implicaciones para el desarrollo local. En J. Gasca y S. Serrano (Coords.), Impactos ambientales, gestión de recursos naturales y turismo en el desarrollo regional (pp. 633-650). Universidad Nacional Autónoma de México y Asociación Mexicana de Ciencias para el Desarrollo Regional A.C.

Arista, L., Hiriart, C. y Barrera-Fernández, D. (2021). Resiliencia y conservación en Pueblos Mágicos de México. Los casos de Pátzcuaro y Mexcaltitán. Bitácora Urbano Territorial, 31(1), 195-210. https://bit.ly/2P2fEy1

Babini, A., Alcaraz, O., Hernández, J. y Salgado, A. (2012). Análisis urbano y morfológico de Taxco de Alarcón, Gro. Siglo XX. Marco teórico metodológico. Topofilia, Revista Científica de Arquitectura, Urbanismo y Territorios, 3(1), 1-38. https://bit.ly/3qB8e2z

Babini, A. y Hernández, J. (2012). Una aproximación al imaginario urbano y social de Taxco de Alarcón. Primeros setenta años del siglo XX. Memoria histórica. Topofilia, Revista Científica de Arquitectura, Urbanismo y Territorios, 3(2), 1-33. https://bit.ly/3qCWzAr

Babini, A. y Hernández, J. (2013). Taxco de Alarcón: magia y realidad. Topofilia, Revista Científica de Arquitectura, Urbanismo y Territorios, 4(3), 1-14. https://bit. ly/3s4x4bC

Balslev, H. y Velázquez-García, M. A. (2010). La posición social y espacial en una ciudad turística: las luchas simbólicas de Álamos, Sonora. PASOS, Revista de Turismo y Patrimonio Cultural, 8(1), 47-59. https://doi.org/10.25145/ j.pasos.2010.08.004

Baños, J., Muñoz, M. y Tovar, R. (2016). Patrimonio y transformaciones en los pueblos mágicos de San Sebastián del Oeste, Jalisco, y Xala, Nayarit, México. Topofilia, Revista Científica de Arquitectura, Urbanismo y Territorios, 9(13), 17-28. https://bit.ly/3pyRVIB

Cazal, A. y Omar, L. (2015). Bacalar, Quintana Roo. Historia y naturaleza para el desarrollo local. En L. López, C. Valverde, A. Fernández y M. Figueroa, (Coords.), Pueblos Mágicos. Una visión interdisciplinaria (Vol. 1, pp. 203-224). Universidad Autónoma Metropolitana - Xochimilco y Universidad Nacional Autónoma de México.

Chavoya, J., Rodríguez, M. y Muñoz, H. (2013). Entre el escenario de la tradición y la emergencia del turismo: Talpa, San Sebastián del Oeste y Mascota. Topo- 
filia, Revista Científica de Arquitectura, Urbanismo y Territorios, 4(1), 761-777. https://bit.ly/37JYLPp

Córdova, K., Guillén, M. y Gutiérrez, V. (2018). Oportunidad laboral para los jóvenes residentes de una ciudad turística: estudio de caso Álamos, Sonora. En J. Enríquez (Coord.) Turismo, sustentabilidad y desarrollo local. Tendencias del desarrollo turístico en una región del noroeste de México (pp. 178-189). Pearson Educación.

Cornejo, J., Andrade, E., Chávez-Dagostino, R. y Espinoza, R. (2018). Percepción de la población local sobre los impactos del turismo en el Pueblo Mágico de Tapalpa, Jalisco, México. PASOS, Revista de Turismo y Patrimonio Cultural, 16(3), 745-754. https://doi.org/10.25145/j.pasos.2018.16.053

Diario Oficial de la Federación (DOF). (2014). Acuerdo por el que se establecen los Lineamientos generales para la incorporación y permanencia al Programa Pueblos Mágicos. https://bit.ly/3bbM4xs

Diario Oficial de la Federación (DOF). (2015). Acuerdo por el que se emiten las Reglas de Operación del Programa de Desarrollo Regional Turístico Sustentable y Pueblos Mágicos. https://bit.ly/2ZsnHGf

Díaz, A. y Osorio, M. (2021). Los imaginarios de los actores sociales del Pueblo Mágico de Ixtapan de la Sal, México. PASOS, Revista de Turismo y Patrimonio Cultural, 19(1), 101-115. https://doi.org/10.25145/j.pasos.2021.19.007

Enríquez, J. (2018). Turismo, sustentabilidad y desarrollo local. Tendencias del desarrollo turístico en una región del noroeste de México. Pearson Educación y Universidad de Sonora.

Enríquez, J., Guillén, M. y Valenzuela, B. (2017). Magdalena de Kino, Sonora. Turismo religioso en un pueblo mágico del noroeste mexicano. En L. López, C. Valverde y M. Figueroa (Eds.), Pueblos Mágicos. Una visión interdisciplinaria (Vol. 3). Universidad Autónoma Metropolitana - Xochimilco y Universidad Nacional Autónoma de México.

Enríquez, J., Guillén, M. y Valenzuela, B. (2018). Patrimonio cultural en los pueblos mágicos. Pearson Educación y Universidad de Sonora.

Enríquez, J. y Guillén, M. (2016). Turismo cultural y comunidad en el pueblo mágico de Álamos, Sonora. Topofilia, Revista Científica de Arquitectura, Urbanismo y Territorios, 9(13), 80-96. https://bit.ly/3dpgW02 
Enríquez, J. y Guillén, M. (2017). Turismo cultural e identidad en el pueblo mágico de Álamos Sonora, México. International Journal of Scientific Management and Tourism, 3(4), 495-512. https://bit.ly/3rV2AbY

Enríquez, J. y Guillén, M. (2018). Patrimonio cultural frente a la turistificación de los pueblos mágicos en el noroeste de México. Topofilia, Revista Científica de Arquitectura, Urbanismo y Territorios, 17, 199-225. https://bit.ly/2N0pUX7

Enríquez, J., Guillén, M., Jaime, M. y Valenzuela, B. (2015). Imaginarios sociales de la comunidad en el pueblo mágico del Fuerte, Sinaloa, México. Imagonautas, 5(1-2), 82-99. https://bit.ly/3rYo47K

Enríquez, J., Vargas, R. y León, C. (2017). Imaginarios Sociales e impactos del turismo residencial en ciudades del noroeste de México. Universidad de Sonora.

Equihua, G., Messina, S. y Ramírez-Silva, J. (2015). Los Pueblos Mágicos: una visión crítica sobre su impacto en el desarrollo sustentable del turismo. Revista Fuente. Nueva Época, 6(22), 1-7. https://bit.ly/3byPjPZ

Fernández, A. (2014). Emociones, identidad, turismo y vida en Huasca (México). Investigaciones Turísticas, 8, 23-45. https://doi.org/10.14198/INTURI2014.8.02

Fernández, A. (2016). Habitar, visitar, sentir, opinar: Huasca de Ocampo. En E. Méndez (Coord.), Pueblos mágicos. Variaciones de tradición y modernidad (pp. 161180). Universidad de Guadalajara.

Fernández, A., Valverde, M. y López, L. (2013). La magia de los pueblos mágicos. Topofilia, Revista Científica de Arquitectura, Urbanismo y Territorios, 4(3), 1-20. https://bit.ly/2ZiKPqC

García, E. y López, V. (2018). Propuesta de una agenda para la investigación del turismo sustentable en los pueblos mágicos de Puebla. Regiones y Desarrollo Sustentable, 18(34), 1-28. https://bit.ly/3tXAbUF

García, A. y Méndez, E. (2018). El relato de la marca pueblos mágicos: una interpretación desde las narrativas del patrimonio arquitectónico. ACE: Architecture, City and Environment, 12(36), 161-176. http://dx.doi.org/10.5821/ace.12.36.4815

García, K. (2017). Cambios en el paisaje urbano de un pueblo mágico. International Journal of Scientific Management and Tourism, 3(4), 75-99. https://bit.ly/3rWSoQ7

Gómez, E., Barrasa, S. y García, A. (2018). Paisaje litoral de la Laguna de Bacalar (Quintana Roo, México): ocupación del suelo y producción del imaginario por 
el turismo. Investigaciones Geográficas, 95, 1-18. https://doi.org/10.14350/ rig.59594

Hernández, J. (2009). Tequila: centro mágico, pueblo tradicional. ¿Patrimonialización o privatización? Andamios, 6(12), 41-67. https://bit.ly/2ZhsU3T

Ibarra, J. y Velarde, M. (2016). El Programa Pueblos Mágicos y la sustentabilidad turística; el caso de Cosalá y los eventos "Cosaltazo" y "Cosalazo". El Periplo Sustentable, 31, 1-22. https://bit.ly/3qvejO6

Landeros, H., Gomora, M. y Castañeda, T. (2015, 20 de noviembre). Enfoques de análisis en pueblos mágicos: ¿eficacia o eficiencia? $20^{\circ}$ Encuentro Nacional sobre Desarrollo Regional en México. Simposio dirigido por la Asociación Mexicana de Ciencias para el Desarrollo Regional y la Universidad Nacional Autónoma de México, Cuernavaca, Morelos.

Lara, M. (2015). El presupuesto participativo como herramienta de inclusión. El programa Pueblos Mágicos. En R. Hernández (Coord.), Pueblos mágicos: discursos y realidades. Una mirada desde las políticas públicas y la gobernanza (pp. 5585). Universidad Autónoma Metropolitana - Lerma y Juan Pablos Editor.

López, L., Valverde, C. y Figueroa, M. (Coords.). (2014). Pueblos Mágicos. Una visión interdisciplinaria (Vol. 1). Universidad Autónoma Metropolitana - Xochimilco y Universidad Nacional Autónoma de México.

López, L., Valverde, C. y Figueroa, M. (Coords.). (2015). Pueblos Mágicos. Una visión interdisciplinaria (Vol. 2). Universidad Autónoma Metropolitana - Xochimilco y Universidad Nacional Autónoma de México.

López, L., Valverde, C. y Figueroa, M. (Coords.). (2017). Pueblos Mágicos. Una visión interdisciplinaria (Vol. 3). Universidad Autónoma Metropolitana - Xochimilco y Universidad Nacional Autónoma de México.

López, L., Valverde, C. y Figueroa, M. (Coords.). (2018). Pueblos Mágicos. Una visión interdisciplinaria (Vol. 4). Universidad Autónoma Metropolitana - Xochimilco y Universidad Nacional Autónoma de México.

Madrid, F. (2014). Gobernanza turística= destinos exitosos: el caso de los pueblos mágicos de México. Universidad Anáhuac México Norte.

Madrid, F. (2019). Derivaciones epistémicas de una política pública: el caso de los Pueblos Mágicos 2001-2015. El Periplo Sustentable, 36, 184-229. https://bit. ly/3bBiCB5 
Manjarrez, J. (2017). Conservación del patrimonio vs turismo cultural: Las ciudades patrimonio y los Pueblos Mágicos. El caso del estado de Puebla (México). En E. Galicia, F. Quiles y S. Ruiz (Eds.), Acervo Mexicano. Legado de Culturas (pp. 108-127). Universidad Pablo Olavide y Benemérita Universidad Autónoma de Puebla.

Martínez, R. y Mínguez, M. (2015). El turismo religioso como elemento revalorizador de una tradición local: la incorporación de Jalostotitlán a los pueblos mágicos. International Journal of Scientific Management and Tourism, 1(2), 53-79. https://bit.ly/3u93jzo

Méndez, E. (2016). El imaginario de la ciudad. Universidad de Guadalajara.

Méndez, E. y Baños, J. (2013). Variantes de lo mexicano: en búsqueda de representaciones arquitectónicas de la autenticidad para el turismo de los pueblos mágicos de Álamos y San Sebastián del Oeste. Topofilia, Revista Científica de Arquitectura, Urbanismo y Territorios, 4(1), 57-73. https://bit.ly/3poHotg

Méndez, E. y Rodríguez, S. (2013). Definiendo “lo mexicano". Una clave: persistencias del modelo urbano colonial en los "pueblos mágicos". Diálogos Latinoamericanos, 14(21), 46-67. https://bit.ly/3rXHIGx

Méndez, E. y Sandoval, Y. (2013). La "magia" puesta en valor: ¿es el mapa de lugares turísticos de los mexicanos una frontera inexpugnable? Topofilia, Revista Científica de Arquitectura, Urbanismo y Territorios, 4(1), 800-819. https://bit. ly/3qvxMhS

Méndez, E. (2012). Re-significación de lugares turístico. Álamos y El Fuerte bajo la intervención del Programa Pueblos Mágicos en 2012. Topofilia, Revista Científica de Arquitectura, Urbanismo y Territorios, 3(2), 1-20. https://bit.ly/2N0qDaN

Méndez, E. (2016). Pueblos Mágicos. Variaciones de tradición y modernidad. Universidad de Guadalajara.

Meza, F., Enríquez, J. y Barrera, F. (2018). Álamos, Sonora, a la sombra del turismo. Entre la magia y la muerte. En J. Enríquez (Coord.), Turismo, sustentabilidad y desarrollo local. Tendencias del desarrollo turístico en una región del noroeste de México (pp. 138-148). Pearson Educación y Universidad de Sonora.

Muñoz, E. (2019). Participación ciudadana y patrimonio cultural en la planificación turística de los pueblos mágicos (México): alcances y limitaciones. Turismo y Sociedad, 25, 29-50. https://doi.org/10.18601/01207555.n25.02 
Noriega, P. (2017). Cadena de consecuencias del Programa Federal Pueblos Mágicos en el mercado de trabajo. El caso de Mazamitla, Jalisco. En P. Pineda (Coord.), Sociedad y gobierno en América Latina. Enfoques, expresiones y problemáticas (pp. 107-124). Universidad de Guadalajara.

Palafox, A., Martínez, M. y Anaya, J. (2016). Nueva ruralidad y sustentabilidad social en el pueblo mágico de Calvillo, Aguascalientes. RITUR, Revista Iberoamericana de Turismo [Número Especial], 6, 64-81. https://doi.org/10.2436/20.8070.01.14

Rodríguez, A. (2012). Entre la memoria y el olvido: recorriendo Álamos en busca de la magia. Topofilia, Revista Científica de Arquitectura, Urbanismo y Territorios, 3(1), 1-13. https://bit.ly/3jPM8XM

Rodríguez, I. (2013). Desafío y dilema en la política pública del "pueblo mágico" mexicano. Topofilia, Revista Científica de Arquitectura, Urbanismo y Territorios, 4(3), 1-22. https://bit.ly/2ZxYQkJ

Rodríguez, J. (2017). Programa de Pueblos Mágicos en localidades turísticas de México. Estudios Jaliscienses, 110, 52-75. https://bit.ly/3snaomW

Rodríguez, S. (2012). Pueblo mágico El Fuerte. Recorrido turístico a partir del imaginario colectivo. Topofilia, Revista Científica de Arquitectura, Urbanismo y Territorios, 3(2), 1-31. https://bit.ly/3jVCIKq

Rodríguez, S. (2014). El montaje de escenarios en El Rosario, Pueblos Mágicos. En A. Valenzuela, C. Alvarado, C. Saldaña y G. Gama (Coords.), Imaginarios del paisaje y el turismo. Entre tradición y distintivos oficiales (pp. 65-84). Juan Pablos Editor y Universidad Autónoma del Estado de México.

Rodríguez, S. y Vargas, R. (2013). Sistema turístico de pueblos mexicanos: Oferta turística a través del programa pueblos mágicos. Topofilia, Revista Científica de Arquitectura, Urbanismo y Territorios, 4(3). https://bit.ly/2NjA8IA

Rojo, S. y Castañeda, M. (2012). Turismo y desarrollo. Caso Álamos, Sonora. Topofilia, Revista Científica de Arquitectura, Urbanismo y Territorios, 3(1), 1-14. https://bit.ly/2NsW9hx

Rojo, S. y Llanes, R. 2009. Patrimonio y turismo: el caso del Programa Pueblos Mágicos. Topofilia, Revista Científica de Arquitectura, Urbanismo y Territorios, 1(3), 1-15. https://bit.ly/3b4XnHM 
Romero, G. (2012). Descripción de la experiencia del recorrido de Álamos. “Un itinerario de fantasmas". Topofilia, Revista Científica de Arquitectura, Urbanismo y Territorios, 3(1), 1-18. https://bit.ly/3rWEZYn

Ruiz, C. (2016). Prácticas cotidianas en la construcción del territorio del pueblo mágico de Tepoztlán, Morelos. Topofilia, Revista Científica de Arquitectura, Urbanismo y Territorios, 9(13), 52-79. https://bit.ly/3bdupp0

Saiz, J. (2018). Turismo sostenible y emprendimiento social. El pueblo mágico de Tequila, México. Retos, Revista de Ciencias de Administración y Economía, 8(15), 51-67. https://doi.org/10.17163/ret.n15.2018.04

Secretaría de Turismo (Sectur). (2020a). Pueblos Mágicos de México. https://bit.ly/ 3b8UF46

Secretaría de Turismo (Sectur). (2020b). Convocatoria para el nombramiento de pueblos mágicos, 2020. Gobierno de México. https://bit.ly/3sakDez

Secretaría de Turismo (Sectur). (2020c). Estrategia Nacional de Pueblos Mágicos. https://bit.ly/37ggL3i

Secretaría de Turismo (Sectur). (2020d). Diagnóstico socioeconómico de los pueblos mágicos. Gobierno de México. https://bit.ly/2N9h3SU

Shaadi, R., Pulido, J. y Rodríguez, I. (2018). La consolidación turística en los territorios que conforman el Programa Pueblos Mágicos (México). Un análisis de sus estrategias competitivas. Investigaciones Turísticas, 15, 1-33. http://dx.doi. org/10.14198/INTURI2018.15.01

Valenzuela, A. (2017). Patrimonio, turismo y mercado inmobiliario en Tepoztlán, México. PASOS, Revista de Turismo y Patrimonio Cultural, 15(1), 181-193. https://doi.org/10.25145/j.pasos.2017.15.011

Valenzuela, A., Saldaña, M. y Vélez, G. (2012). Identidad, estructura barrial y control social del espacio en Tepoztlán, Morelos. Topofilia, Revista Científica de Arquitectura, Urbanismo y Territorios, 3(1), 1-25. https://bit.ly/3rTIEGv

Valenzuela, B., Campa, R. y López, M. (2012). Factores psicosociales asociados al imaginario social a partir de las percepciones de niños y jóvenes en el Fuerte, Sinaloa. Topofilia, Revista Científica de Arquitectura, Urbanismo y Territorios, 3(2), 1-20. https://bit.ly/2Zng6sy 
Valverde M. y Enciso, J. (2013). La magia de los pueblos: ¿atributo o designación? Turismo cultural en México. Academia XXII, 4(7), 11-25. http://dx.doi.org/ 10.22201/fa.2007252Xp.2013.7.43000

Vargas, A. y Rodríguez, I. (2014). Dinámica relacional de la gestión turística en el pueblo mágico de Calvillo, Aguascalientes, México. Teoría y Praxis, 14, 137160. https://doi.org/10.22403/UQROOMX/TYPNE2014/05

Vargas, R. y Enríquez, J. (2015). Configuración de los imaginarios para la promoción turística en Álamos, Sonora. Topofilia, Revista Científica de Arquitectura, Urbanismo y Territorios, 5(1), 1-17. https://bit.ly/3rQZENs

Vázquez, C. A.y Díaz, O. F. (2015). La participación ciudadana en el Programa Pueblos Mágicos desde el enfoque de la gobernanza y las redes de políticas públicas. Unestudio sobre lavinculación entregobierno, actores público-socialesy privados en el caso de Valle de Bravo. En R. Hernández (Coord.), Pueblos mágicos: discursos y realidades. Una mirada desde las políticas públicas y la gobernanza (pp. 199-247). Universidad Autónoma Metropolita - Lerma y Juan Pablos Editor.

Velázquez-García, M. A. y Aragón, M. (2015). Todos Santos, Baja California Sur. La experiencia de lo mexicano-americano: las misiones y el Hotel California. En L. López, C. Valverde y M. Figueroa (Coords.), Pueblos Mágicos. Una visión interdisciplinaria (Vol. 2, pp. 227-248). Universidad Autónoma Metropolitana - Xochimilco y Universidad Nacional Autónoma de México.

Velázquez-García, M. A. y Labra, A. (2015). Logros y problemáticas de los Pueblos Mágicos del Estado de Hidalgo. Revista Semestral de Estudios Regionales, 2(4), 46-60. https://bit.ly/3ugicJ3

Warnholtz, G. (2014). El programa Pueblos Mágicos en el laberinto de las políticas públicas mexicanas. En A. Valenzuela, C. Alvarado, C. Saldaña y G. Gama (Coords.), Imaginarios del paisaje y el turismo. Entre tradición y distintivos oficiales (pp. 289-307). Juan Pablos Editor y Universidad Autónoma del Estado de México.

Zárate, M., Almejo, A., Ayala, E. y Pitones, J. (2018). Paisaje y patrimonio de un pueblo mágico fronterizo. Topofilia, Revista Científica de Arquitectura, Urbanismo y Territorios, 9(17), 131-146. https://bit.ly/2ZIX2Lu 